\title{
Levantamento bibliométrico sobre o desenvolvimento de produtos inovadores e sustentáveis
}

\author{
Bibliometric Survey on the development of innovative and sustainable products
}

\author{
Deloísa Pereira Burin ${ }^{1}$ \\ (D) Caroline Butzge ${ }^{2}$ \\ Milena Fontoura da Silva ${ }^{3}$ \\ Jorge André Ribas Moraes ${ }^{4}$ \\ Daniel Augusto Hoppe ${ }^{5}$
}

\section{Resumo}

A pesquisa teve como objetivo apurar o estado da arte no quesito inovação e sustentabilidade em projetos de produtos. Portanto, identificou-se os artigos publicados na base de dados Scopus (Elsevier) e fez-se um estudo bibliométrico sobre o tema planejamento e desenvolvimento de produtos sustentáveis. Tratase de uma pesquisa exploratória, cujo período analisado foi de 2007 a 2017. Foram encontrados 147 artigos publicados abordando os termos innovation, project management, product development e sustainability. Através de uma análise quantitativa dos resultados, verificou-se um aumento gradativo de publicações a partir de 2012. Em relação aos países de origem dessas publicações, o Brasil encontra-se em 4ㅇ lugar, o que evidencia sua preocupação em relação ao desenvolvimento de produtos de cunho sustentável. Utilizou-se também o software VOSviewer para identificação das palavras-chave com maior ocorrência e os autores que mais publicaram em relação ao conjunto de termos.

${ }^{1}$ Universidade de Santa Cruz do Sul Graduada em Engenharia de Produção Santa Cruz do Sul, Rio Grande do Sul, Brasil hburin@m×2.unisc.br

${ }^{2}$ Universidade de Santa Cruz do Sul Graduada em Engenharia de Produção Santa Cruz do Sul, Rio Grande do Sul, Brasil carolinebutzge@m×2.unisc.br

${ }^{3}$ Universidade de Santa Cruz do Sul Graduada em Engenharia de Produção Santa Cruz do Sul, Rio Grande do Sul, Brasil milenafontoura@mx2.unisc.br

${ }^{4}$ Universidade de Santa Cruz do Sul Doutor em Engenharia de Produção Coord. Curso de Especialização em Engenharia de Segurança do Trabalho - 8 ed. Coord. Adjunto do Programa de Mestrado e Doutorado em Tecnologia Ambiental Santa Cruz do Sul, Rio Grande do Sul, Brasil jorge@unisc.br

${ }^{5}$ Universidade de Santa Cruz do Sul Mestre em Engenharia de Produção Santa Cruz do Sul, Rio Grande do Sul, Brasil danielhoppe@unisc.br
Palavras-chave: Sustentabilidade. Inovação. PDP. Gestão de Projetos.

\section{Abstract}

The research aimed to investigate state of the art in terms of innovation and sustainability in projects. Therefore, articles published in the Scopus (Elsevier) database were identified and a bibliometric study about the theme of sustainable product planning and development was conducted. This is an exploratory research, whose period analyzed was from 2007 to 2017. There were found 147 articles published approaching the terms "innovation", "project management", "product development" and "sustainability". Through a quantitative analysis of the results, there was a gradual increase of publications from 2012. To the countries of origin of these publications, Brazil is in 4th place, which shows its concern regarding sustainable products development. Furthermore, VOSviewer software was used to identify the most frequently occurring keywords and the most published authors to the set of terms.

Keywords: Sustainability. Innovation. NPD. Project Management. 
1 Introdução

Questões de cunho sustentável são atualmente os maiores desafios que toda sociedade enfrenta (Dobrovolskienè \& Tamošiūnienè, 2015). É importante que as mudanças em relação ao desenvolvimento sustentável ocorram a nível global, com constante apoio governamental principalmente às organizações, para que tenham condições de crescer no caminho da sustentabilidade (Gmelin \& Seuring, 2014).

É preciso que as empresas percebam que as práticas sustentáveis não são custos adicionais, mas oportunidades de aumentar a sua competitividade frente ao mercado (Albino, Balice, \& Dangelico, 2009). Uma ação que vem ganhando força é o desenvolvimento de produtos sustentáveis (Dangelico, 2016), porém, é um grande desafio conseguir integrar características de sustentabilidade a novos projetos inovadores. Nesse sentido, a inovação é considerada de grande valia em resposta às rápidas mudanças do mercado (Agyapong, Agyapong, \& Poku, 2017).

Carvalho, Silvestre e Cunningham (2017) salientam a crescente preocupação das empresas no que tange à inovação de produtos mais sustentáveis, os quais são produzidos, por exemplo, com menos energia ou com a substituição de matérias-primas que agridem o meio ambiente, tornando-se possível sua reciclagem. Além disso, outras atitudes podem ser colocadas em prática, levando a vantagens competitivas frente aos concorrentes. Podese citar a destinação adequada de seus resíduos, a criação de fontes próprias de energia, a diminuição de desperdícios internos, a preocupação com a origem de suas matérias-primas, dentre outras iniciativas.

Devido às constantes mudanças no cenário internacional, as organizações estão sendo obrigadas a inovarem, modificando e desenvolvendo produtos com apelos sustentáveis (Martens, Kniess, Martens, \& Carvalho, 2016). Tais inovações são alternativas para que as empresas se mantenham competitivas no mercado (Bocken et al., 2014). Além disso, Dangelico (2016) salienta que as empresas ganham em redução de custos, vantagens de mercado, boa reputação frente à sociedade e grandes possibilidades de inovação.

Capacidades inovadoras impulsionam a competição, afetando custos e diferenciação no portfólio de produtos (Carayannis \& Grigoroudis, 2012). Quando correlacionadas com sustentabilidade, leva-se em consideração os princípios econômicos, ambientais e sociais, o chamado tripé da sustentabilidade (Martens et al., 2016).

Dobrovolskienė e Tamošiūnienè (2016) afirmam que os termos sustentabilidade e gerenciamento de projetos foram relatados recentemente na literatura. Nesse sentido, novos conceitos devem ser incorporados em relação a essas práticas (Brones, Carvalho, \& Zancul, 2014), cabendo às organizações desenvolverem métodos eficientes de gerenciamento com possibilidades sustentáveis (Martens et al., 2016). Sustentabilidade atrelada à gestão de projetos tem como foco fortalecer a economia, preservar os recursos naturais e impactar positivamente a sociedade (Dobrovolskienè \& Tamošiūnienè, 2015).

Um fator considerável para que as empresas adotem essas medidas é a constante evolução do mercado, onde os clientes estão em busca de soluções e produtos que atendam ao apelo ambiental (Dangelico, 2016). Em contrapartida, isso gera um considerável aumento de custos para as empresas, como investimentos em Planejamento e Desenvolvimento de Produtos (PDP), gerenciais e operacionais, cabendo às organizações 
administrar esse aumento e criar estratégias de preços para atrair os consumidores (Carvalho et al., 2017). O setor de PDP, embora complexo, é essencial às empresas, pois garante qualidade aos produtos formalizando as etapas (Kechinski, Faccio, Rosa, \& Echeveste, 2010).

Carvalho et al. (2017) salientam que ao investirem em produtos inovadores e sustentáveis, é preciso que as organizações entendam o que leva os consumidores a adquirir essas inovações. Ideias internas podem ser levadas em consideração para o PDP, mas é essencial uma análise do ambiente externo em busca de ideias e sugestões (Pihlajamaa, Kaipia, Säilä, \& Tanskanen, 2017). Kahn, Barczak, Nicholas, Ledwith e Perks (2012) enfatizam ainda que uma pesquisa de mercado é realizada no intuito de conhecer os clientes, concorrentes e, consequentemente, o mercado.

O desenvolvimento de produtos sustentáveis tem como objetivo atender as necessidades e desejos dos clientes finais, além de prezar pela diminuição dos seus impactos ambientais e sociais, tudo isso atrelado à geração de valor econômico para as empresas (Gmelin \& Seuring, 2014). É fundamental que sejam feitas pesquisas de mercado no intuito de dar atenção aos consumidores, buscar entender seus anseios, para assim adotar as melhores estratégias de inovação (Dangelico, 2016). Theis, Haubert, Zmijewski e Schreiber (2017) evidenciam que a inovação é capaz de atender às demandas e expectativas dos consumidores.

Há, no entanto, pouco entendimento das ferramentas e técnicas utilizadas nos projetos que levam à inovação ambiental, tornando-se, assim, um desafio para as organizações (Bocken, Farracho, Bosworth, \& Kemp, 2014). Conseguir compreender esses desafios e buscar meios para mitigá-los pode significar possibilidades de grandes ganhos para as empresas, tornando-as sustentáveis, conquistando novos clientes, desenvolvendo capacidades de competitividade e produtos inovadores.

No intuito de analisar a abordagem dessas questões no meio científico, esta pesquisa buscou, através de uma estudo bibliométrico, investigar o estado da arte na base de dados Scopus (Elsevier), os artigos científicos publicados em um período de dez anos (2007 a 2017), a fim de identificar o número de trabalhos publicados, os países que mais publicam sobre o tema, as áreas, os autores e as palavras-chave que mais aparecem correlacionadas com os termos propostos para tal pesquisa: inovação, sustentabilidade, gestão de projetos e desenvolvimento de produto.

De forma análoga, Morioka e Carvalho (2016) revisaram sistematicamente a literatura publicada entre 1991 e 2011, que aborda gestão de projetos e sustentabilidade de forma simultânea na base ISI Web of Knowledge (Web of Science). Para tanto, foram utilizadas técnicas de bibliometria, redes e análise de conteúdo, e os softwares Sitkis e Ucinet. Em seu estudo, os autores constataram uma vertente da inovação aliada à sustentabilidade, visto que identificou-se uma forte relação entre as palavras-chave inovação, sucesso, desempenho, desenvolvimento de produto e design.

Luiz, Jugend, Jabbour, Luiz, \& Souza (2016), por sua vez, abordaram a evolução dos estudos científicos no que tange ao ecodesign, mapeando os trabalhos mais relevantes dos últimos 20 anos na plataforma Scopus. Percebeu-se, por meio dessa pesquisa, um crescimento na quantidade de publicações sobre esse tema ao longo do período analisado e determinada dispersão sobre a autoria dos artigos, o que dificulta a identificação dos principais autores. Analisando as revistas que publicam a respeito do ecodesign, os pesquisadores verificaram que 
grande parte delas são das áreas de conhecimento relacionadas ao meio ambiente, em oposição aos periódicos que tratam dos termos de desenvolvimento de novos produtos, inovação e gestão de projetos.

Assim como os autores Luiz et al. (2016), na presente pesquisa utilizou-se a ferramenta VOSviewer. Esse software é utilizado para a construção e visualização de redes bibliométricas, baseando-se na distância entre os nós da rede gerada, apontando a intensidade de relação entre eles, sendo ela tão maior quanto forem as distâncias (Van Eck \& Waltman, 2014).

Optou-se por utilizar a plataforma Scopus (Elsevier) devido à sua importância no meio científico e a sua gama de periódicos vinculados. A amostra de artigos encontrados no estudo pode ser apontada como uma representação do estágio de pesquisas envolvendo inovação e sustentabilidade a nível global. Acredita-se que esses dados possam ser um reflexo real de como as organizações têm mudado ao longo dos anos para alcançar melhores resultados competitivos através da união desses conceitos.

Morioka e Carvalho (2016) apontam que há um potencial expressivo a ser explorado no cruzamento entre os conceitos de sustentabilidade e gestão de projetos. De maneira complementar e mais específica, a pesquisa busca examinar a relação entre os termos utilizados pelos autores supracitados, a inovação e o desenvolvimento de produtos, utilizando outra base científica de dados.

Esse estudo permite uma avaliação efetiva da representatividade dos assuntos no meio científico no que tange às áreas de conhecimento e aos países de origem das publicações. As palavras-chave mais frequentes nos trabalhos analisados são destacadas na presente pesquisa, bem como os autores com maior quantidade de publicações abordando os termos destacados. Tanto palavras-chave quanto autores são apresentados pela formação de clusters de modo a expor as ligações entre si.

Além disso, esse trabalho abrange artigos publicados até o ano de 2017, o que caracteriza uma abordagem mais recente se comparado aos demais estudos. Analisando os resultados obtidos, observa-se uma tendência para os próximos anos de aumento no número de publicações que abordam esses temas na plataforma Scopus (Elsevier).

Identificou-se, no desenvolvimento dessa pesquisa, a oportunidade de apresentar à comunidade acadêmica o estado da arte entre os anos de 2007 a 2017 em relação aos termos "inovação", "gestão de projetos", "desenvolvimento de produto" e "sustentabilidade" na Scopus, base de dados de grande impacto no ambiente científico. Por meio dela, busca-se contextualizar a situação nos últimos anos a respeito das publicações que abordam as temáticas citadas, evidenciando sua importância aos pesquisadores.

\section{Metodologia}

A metodologia trata dos procedimentos adotados para se desenvolver uma pesquisa (Barros \& Lehfeld, 2008) e validar as etapas traçadas para atingir os objetivos propostos (Gerhardt \& Silveira, 2009). A metodologia de pesquisa deste estudo está apresentada em forma de um organograma elaborado pelos autores e representada pela Figura 1. As suas etapas, assim com suas características, estão descritas na sequência. 


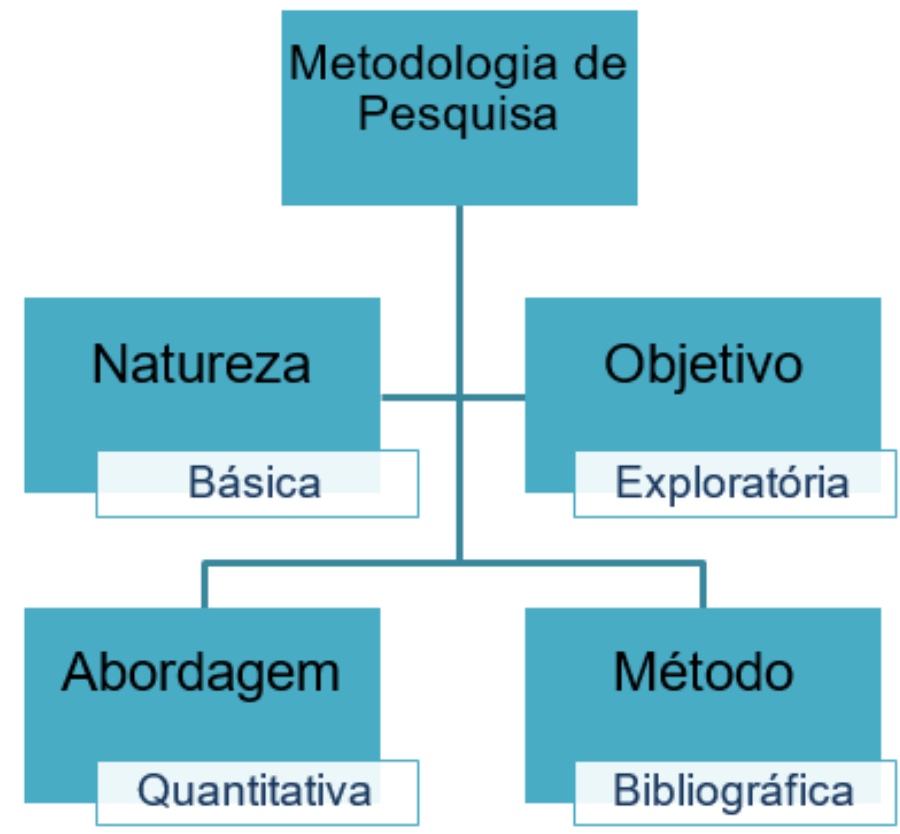

Fonte: Elaborado pelos autores.

A metodologia deste estudo quanto à sua natureza pode ser classificada como básica, visto que não terá aplicação prática, objetivando apenas gerar novos conhecimentos. Em relação aos objetivos, enquadra-se como sendo uma pesquisa exploratória, a qual normalmente envolve pesquisas bibliométricas e é realizada no intuito de proporcionar uma visão ampla do assunto pesquisado (Gil, 2008) ou serve de base para aprofundar os temas (Berto \& Nakano, 2014).

A abordagem quantitativa trata-se da mensuração das variáveis da pesquisa, não havendo interferência do pesquisador sobre elas (Miguel et al., 2010). É utilizada quando se pretende avaliar um entendimento acerca de um fenômeno (Berto \& Nakano, 2014), transformando opiniões ou informações em números para posterior análise e interpretação (Moresi, 2003).

O método de pesquisa utilizado para atender ao objetivo é a pesquisa bibliográfica, onde foi possível analisar os conceitos e publicações a respeito do tema proposto. Esta pesquisa foi realizada levando-se em consideração materiais já elaborados por outros pesquisadores, composto de livros, artigos científicos (Gil, 2008), jornais e redes eletrônicas (Moresi, 2003). Essa análise é importante para compreender os conceitos já publicados (Barros \& Lehfeld, 2008).

O procedimento metodológico, que indica os passos a serem seguidos para o desenvolvimento do estudo e obtenção dos resultados, foi dividido em cinco etapas sequenciais que estão representadas pela Figura 2. Salienta-se que essas são de suma importância para a obtenção dos objetivos propostos. 


\section{Etapa 1 Definição do tema da pesquisa}

\section{Etapa 2 Definição da plataforma de busca}

\section{Etapa 3 Processo de mineração dos dados biliométricos}

\section{Etapa 4 Análise dos dados}

\section{Etapa 5 Conclusão}

Fonte: Elaborado pelos autores.

A primeira etapa do estudo foi a definição do tema a ser pesquisado - o desenvolvimento de produtos inovadores e sustentáveis - escolhido por ser um assunto relativamente novo e com grande importância tanto para a sociedade quanto para o meio acadêmico. A segunda etapa compreende a escolha da base de dados para o levantamento bibliográfico. Optou-se pela plataforma Scopus (Elsevier) por ser de grande representatividade, possuir vínculo com muitos periódicos e, consequentemente, disponibilizar um grande número de artigos para pesquisas.

Para a terceira etapa, a mineração de dados, definiu-se os seguintes termos de busca: innovation, "project management", "product development" e sustainability, traduzidos respectivamente para o português, como: inovação, gerenciamento de projetos, desenvolvimento de produtos e sustentabilidade.

A pesquisa teve como limitações para a busca dos artigos na base de dados Scopus apenas os documentos classificados como sendo artigos que continham as palavras-chave descritas em seu título, resumo ou palavraschave. Além disso, o período considerado foi o de dez anos (2007 a 2017). Essas limitações foram necessárias para que apenas os artigos relacionados diretamente com o tema proposto fossem selecionados para posterior análise.

Primeiramente pesquisou-se, na base já mencionada, apenas o termo "innovation" com as limitações. Na sequência, fez-se um afunilamento dos artigos encontrados, com a introdução dos demais termos gradativamente, na seguinte ordem: "project management", "product development" e "sustainability", resultando em um total de 147 artigos científicos encontrados e analisados.

Através da análise dos dados, na quarta etapa, realizou-se um mapeamento do estado da arte do tema proposto, sendo possível averiguar o número de publicações anuais, os países, as áreas de pesquisa com mais publicações, a relação de palavras-chave mais citadas, bem como os autores que mais publicaram sobre o assunto. Além disso, identificou-se entre os artigos os "gaps" relacionados ao assunto pesquisado. 
Utilizou-se o software VOSviewer para expor as ligações entre as palavras-chave mais citadas nas publicações analisadas e sua frequência de citações. Configurou-se o software para exibir os termos citados mais de 5 vezes, resultando na formação de clusters. O VOSviewer também foi aplicado de maneira a apontar os autores com maior quantidade de publicações que abordam os termos citados. Como resultado, obteve-se treze autores compondo a rede de oito clusters.

Por fim, a quinta etapa aborda as conclusões do trabalho. Nela é feita um fechamento do estudo proposto, apontando as vantagens para as organizações quanto ao desenvolvimento de produtos inovadores e sustentáveis. Também são expostas as considerações finais sobre os resultados encontrados, as sugestões de trabalhos futuros para a sequência do estudo e, por fim, as limitações as quais o trabalho se restringe.

\section{Resultados}

A partir da busca realizada na base de dados Scopus (Elsevier) pôde-se identificar um total de 147 artigos científicos publicados abordando os termos innovation, "project management", "product development" $e$ sustainability, no período de dez anos (2007 a 2017). A Figura 3 apresenta os resultados quantitativos do número de publicações em relação ao seu devido ano. Observa-se um aumento gradativo a partir de 2012, sendo que nesse período o número máximo de publicações foi de 9 artigos, passando para um total de 37, no ano de 2017.

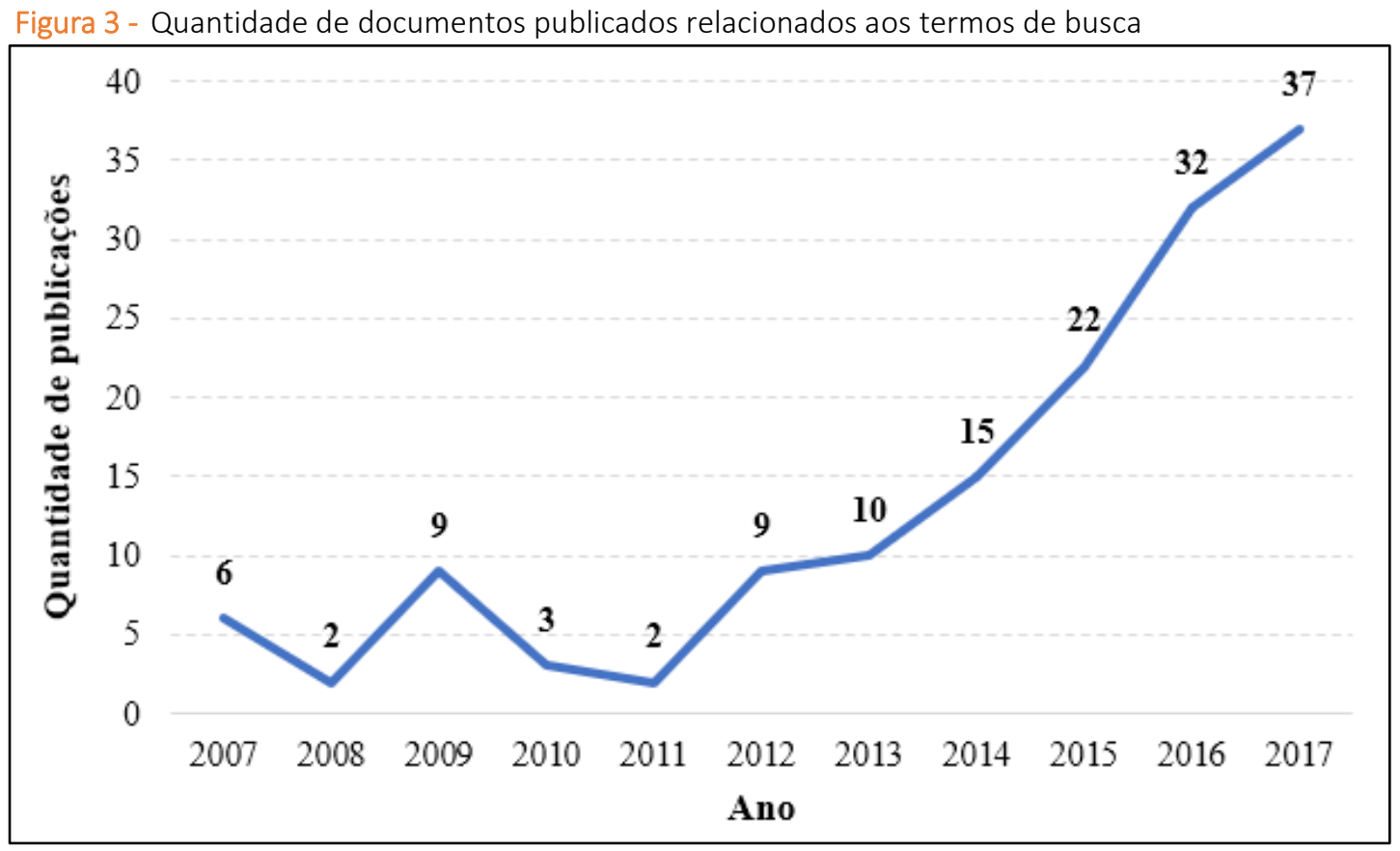

Fonte: Elaborado pelos autores.

Ao analisar a Figura 3, percebe-se um considerável aumento de publicações entre os anos de 2012 a 2017. A justificativa para esse aumento de publicações pode ser reflexo da crescente preocupação das 
organizações em desenvolver produtos inovadores e sustentáveis. Mais de $70 \%$ do total de artigos acerca do tema foram publicados entre 2014 e 2017.

Em relação ao país de origem das publicações, a Figura 4 representa o total de 13 países, os quais foram selecionados pela pesquisa por serem os mais representativos em relação aos termos mencionados anteriormente. Os demais, possuem entre 1 e 6 registros.

Figura 4 - Publicações por países com relação aos termos

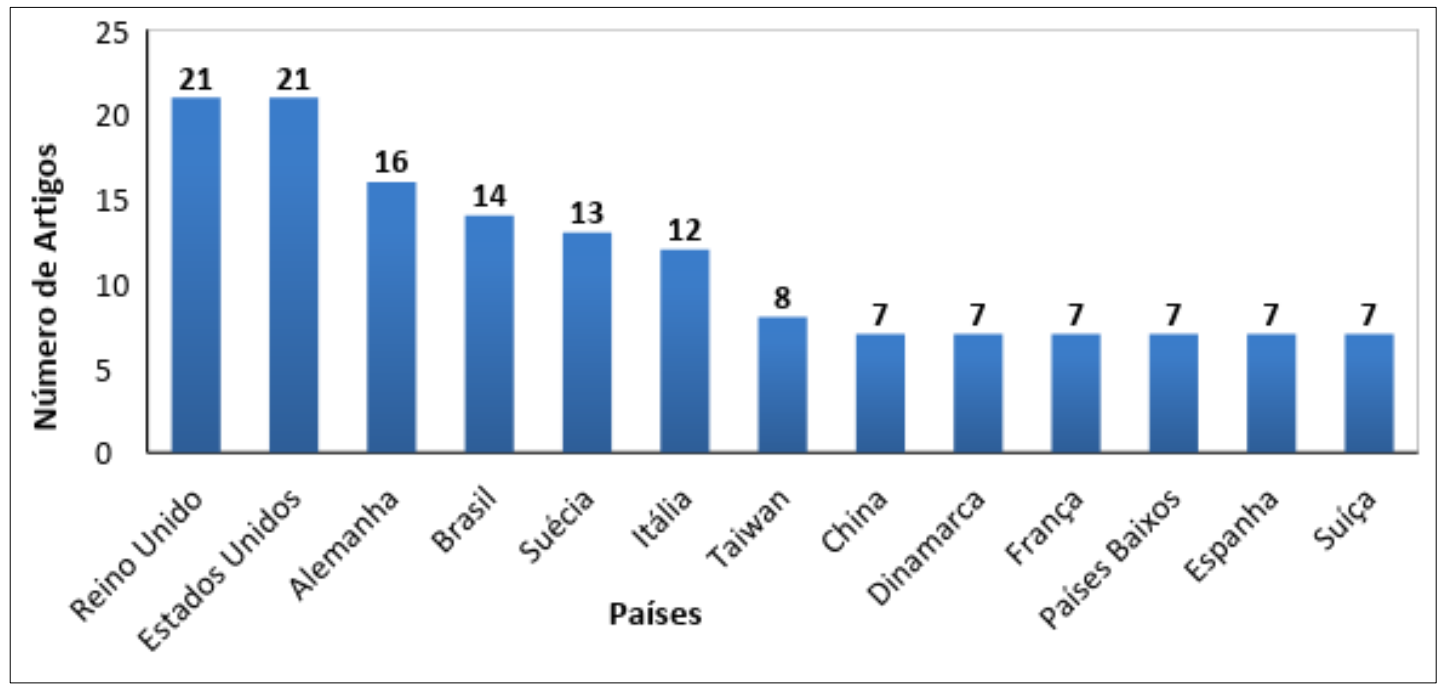

Fonte: Elaborado pelos autores.

Os países responsáveis pelo maior número de publicações são o Reino Unido e os Estados Unidos, ambos com 21 artigos. Abedini, Rahman, Naeini \& Ebrahim (2017), em sua análise bibliométrica a respeito do design industrial, obtiveram os mesmos como os países com maior número de publicações sobre o tema estudado, o que indica seu potencial diante de temas diversos. Os países citados, juntamente com a Alemanha, representam 40\% do total das publicações encontradas na presente pesquisa. Destaca-se o Brasil em quarto lugar com 14 publicações, mostrando que, embora ainda seja um número pequeno de registros, há uma preocupação em relação ao desenvolvimento de produtos inovadores e sustentáveis no Brasil.

Objetivando identificar as áreas de conhecimento nas quais as revistas estão enquadradas, elaborou-se a Figura 5. 
Figura 5 - Publicações por área de conhecimento

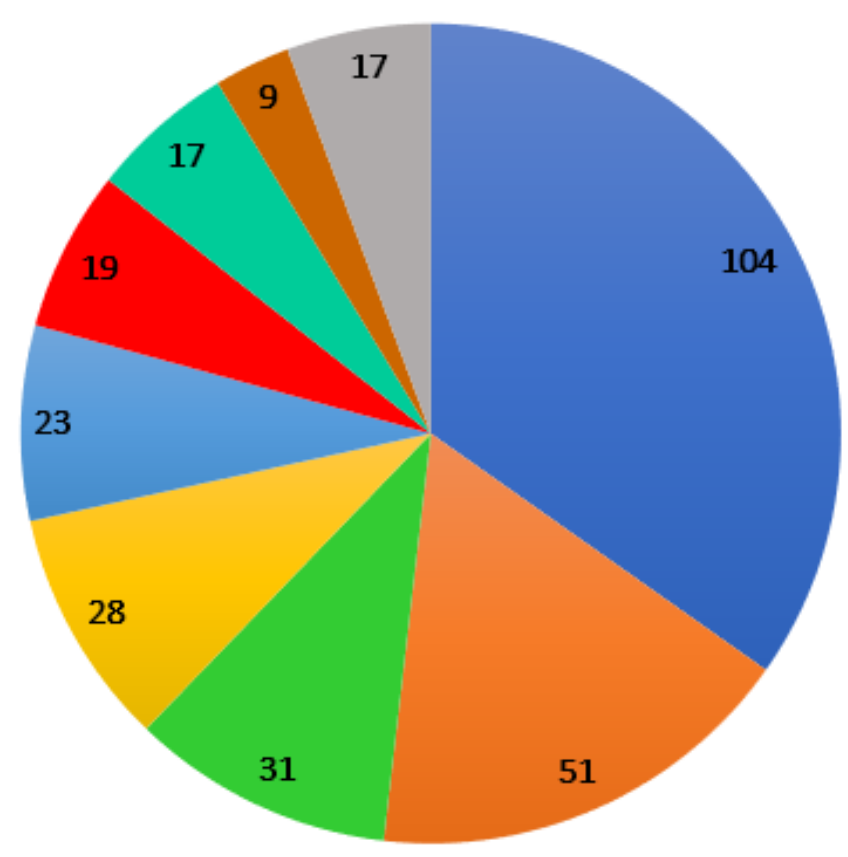

- Negócios, Administração e Contabilidade

Engenharia

Ciências Sociais

— Ciência Ambiental

Energia

- Ciência da Computação

n Ciências da Decisão

- Economia, Econometria e Finanças

- Outras

Fonte: Elaborado pelos autores

A figura 5 evidencia que a área com a maior quantidade de publicações é a de "Negócios, Administração e Contabilidade" com 104 artigos, o que representa aproximadamente 70\% sobre o total. A área denominada "Outras", na Figura 5, representa o aglomerado das demais áreas que continham de 1 a 8 publicações.

Utilizou-se o software VOSviewer para identificação das palavras-chave com maior ocorrência nos 147 artigos analisados, como demonstrado na Figura 6. Criou-se um mapa levando-se em consideração os termos de busca já mencionados, bem como a base de dados e o período. O mapa representa a relação entre as 20 palavraschave identificadas e a densidade de cada círculo representa a frequência de citação. 
Figura 6 - Palavras-chave com maior ocorrência nos 147 artigos analisados

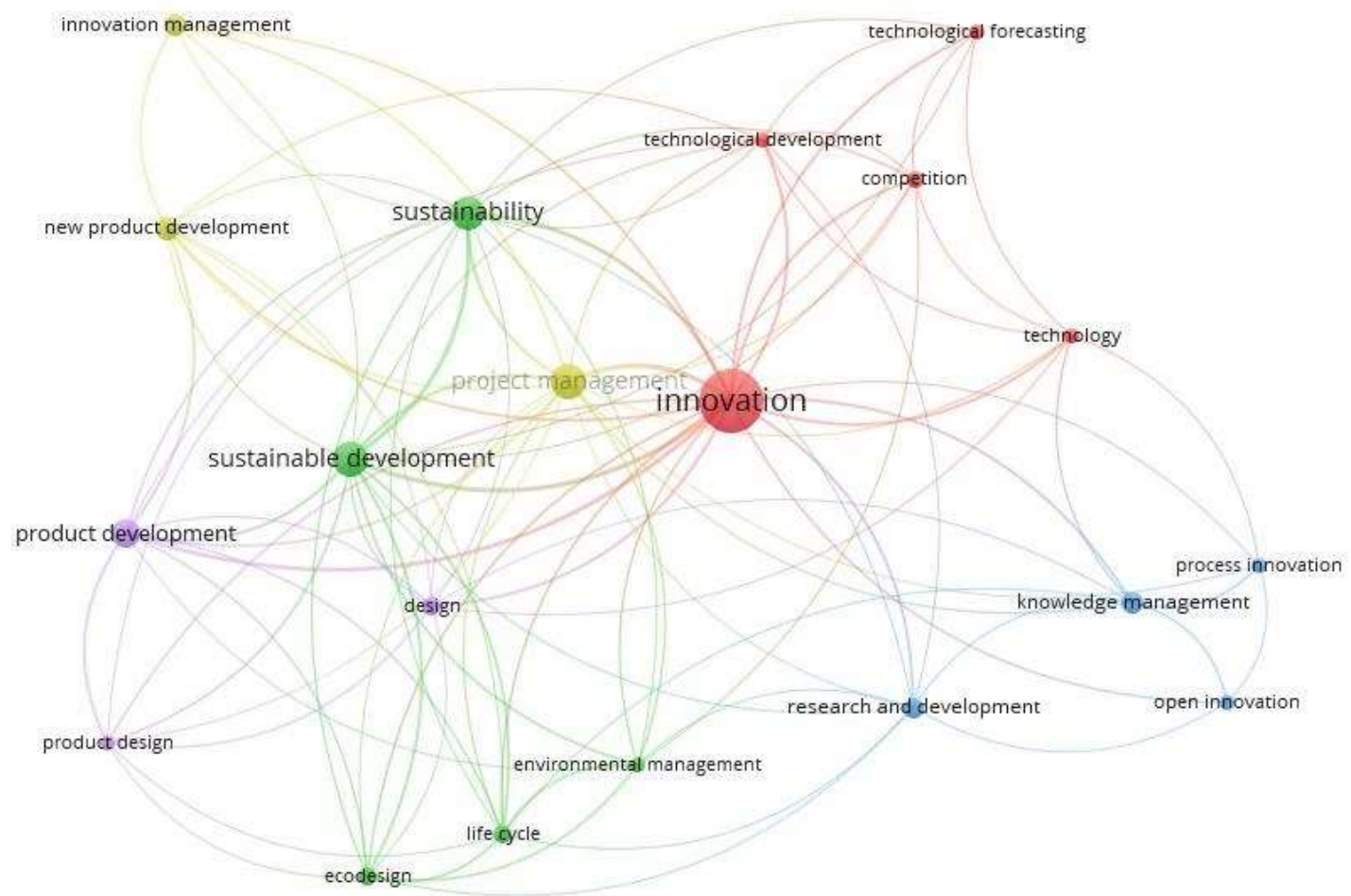

Fonte: Software VoSviewer.

Foram gerados cinco clusters de ligação, representados na Figura 6, por cores, sendo que o primeiro abrange cinco palavras-chave, como segue: competition, innovation, technological development, technological forecasting e technology; o segundo também contém cinco termos: ecodesign, environmental management, life cycle, sustainability e sustainability development; no terceiro cluster, mais quatro termos: knowledge management, open innovation, process innovation e research and development; no quarto, três termos: innovation management, new product development e project management; por fim, no quinto cluster, as três palavras-chave: design, product design e product development. Através desse tipo de análise é possível identificar quais os termos e conceitos que se relacionam com os adotados pela pesquisa, possibilitando uma maior restrição de artigos conforme o que se pretende pesquisar.

Além da análise de ocorrência das palavras-chave, o software VOSviewer foi utilizado para identificar os autores que mais publicam em relação ao conjunto dos termos: innovation, "project management", "product development" e sustainability. Oito clusters foram gerados, representados na Figura 7, por cores para sua diferenciação. 
Figura 7 - Análise dos principais autores com os termos de busca

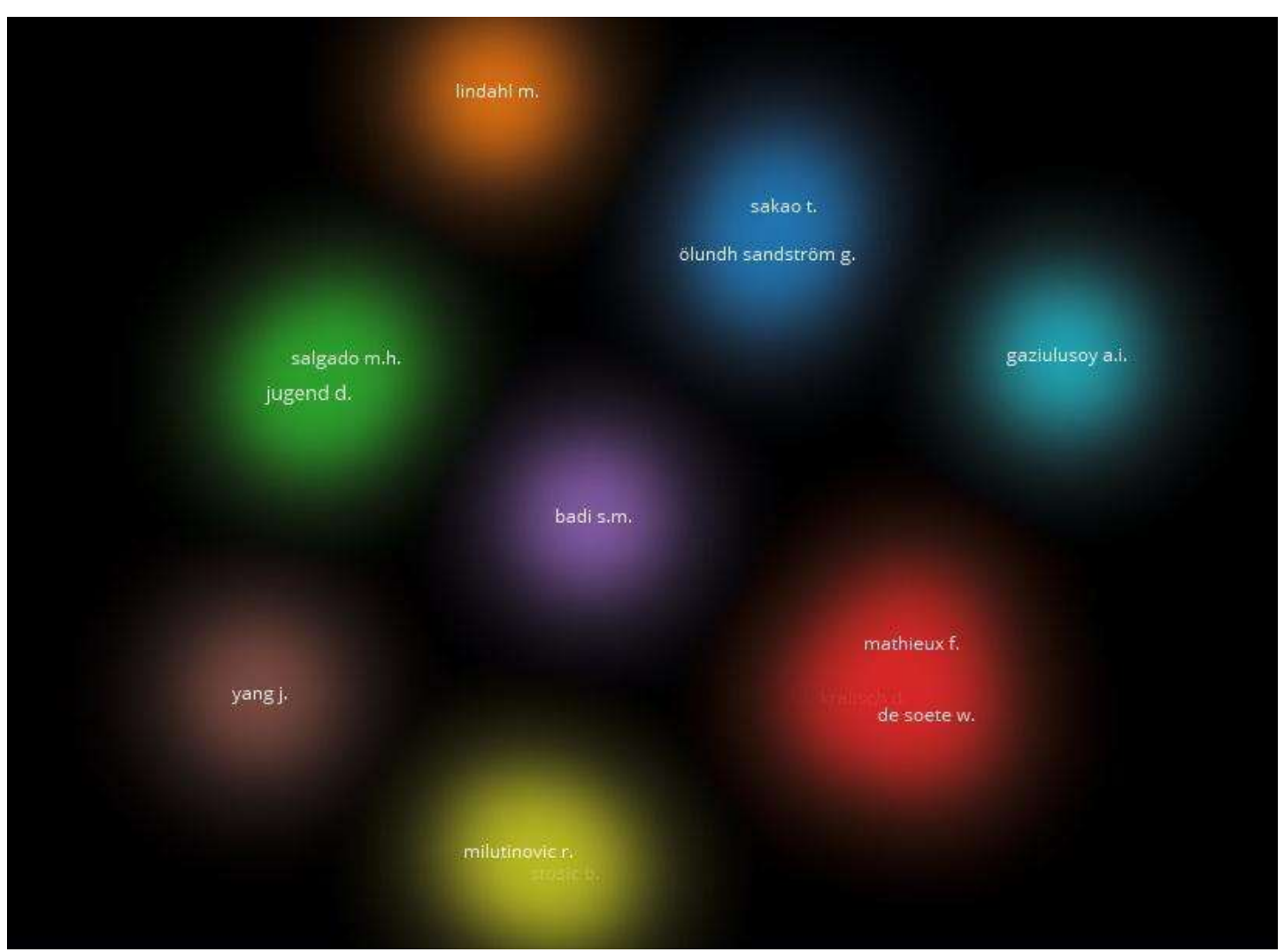

Fonte: Elaborado pelos autores.

Ao total foram identificados treze autores. O primeiro cluster abrange De Soete W., Kralisch D. e Mathieux F.; o segundo, os autores Jugend D. e Salgado M. H.; o terceiro mais dois autores, Sakao T. e Olundh Sandstrom G.; o quarto cluster também apresenta dois autores, Milutinovic R. e Stosic B.; o quinto, sexto, sétimo e oitavo clusters estão representados por apenas um autor, citados respectivamente, Badi S. M., Gaziulusoy A. I., Lindahl M. e Yang J.

Com uma investigação mais criteriosa desses autores, destacam-se os três com maior número de citações de seus documentos, bem como o número de publicações. Em primeiro está o autor alemão Kralisch D. com 1.170 citações, possuindo 65 artigos. Em segundo, o sueco Sakao T. com 1.008 citações e 101 publicações. Por fim, em terceiro tem-se o italiano Mathieux F. com 53 artigos na Scopus e citado por outros 435 documentos. O restante dos autores possui menor número de artigos, com média de 13 publicações e 84 citações por outros autores. Destaca-se que, dentre os 13 autores apontados pelo software VOSviewer, Jugend D. e Salgado M. H. são brasileiros cujos artigos possuem grande impacto no meio científico, sendo citados por 130 e 182 documentos, respectivamente. 
4 Conclusões

Levando-se em consideração as crescentes pressões ambientais, como a economia de energia, de água, redução de desperdícios, substituição de materiais, reuso e reciclagem, é fundamental que as empresas desenvolvam uma consciência ambientalmente correta, passando uma imagem de comprometimento e preocupação para com a sociedade. O desenvolvimento de produtos inovadores e sustentáveis apresenta-se como oportunidades para as organizações adquirirem vantagens competitivas, através de conquistas de novos mercados, clientes e crescimento organizacional. Para que esses produtos sejam aceitos e gerem lucros para a empresa, é importante o desenvolvimento de pesquisas de mercado ainda nas fases de planejamento, para assim identificar o que o cliente realmente deseja, suas necessidades e se ele está disposto a pagar por essa inovação.

Fundamentado no exposto, esta pesquisa foi desenvolvida com o objetivo de investigar o estado da arte relativa a inovação e sustentabilidade em projeto e desenvolvimento de produtos. Utilizando a base de dados Scopus (Elsevier) com a limitação do período de dez anos (2007 a 2017), identificou-se a quantidade de artigos publicados, os países que mais publicaram, as áreas de conhecimento, os autores e as palavras-chave relacionadas aos termos propostos. Para Azarias, Coutinho \& Campos (2018), análises bibliométricas tornam possível conhecer os padrões de determinada literatura e delinear um caminho para desenvolver a pesquisa a partir de uma análise quantitativa.

A pesquisa está voltada para artigos que se restringem às palavras-chave propostas, ou seja, a correlação entre os termos no intuito de identificar as publicações que realmente tratam do assunto de desenvolvimento de produtos inovadores e de cunho sustentável. De maneira complementar, buscou-se examinar a relação entre esses termos utilizados pelos autores Morioka e Carvalho (2016) e Luiz et al. (2016), a inovação e o desenvolvimento de produtos. O presente estudo abrange até o ano de 2017, o que caracteriza uma abordagem mais recente se comparado a esses estudos.

Com os resultados obtidos, foi possível constatar um significativo aumento de publicações sobre o tema entre os anos de 2012 a 2017, o que implica na tendência para os próximos anos de um incremento no número de publicações nessa plataforma que abordam os temas relacionados. Pôde-se perceber, também, que o Brasil é um dos países com interesse nessas questões, ficando em quarto lugar no número de artigos publicados.

Além disso, com a análise realizada no VOSviewer, identificou-se os autores que mais pesquisam sobre o tema e as relações entre eles, bem como as palavras-chave mais citadas nos 147 artigos encontrados acerca do tema de pesquisa: a com maior densidade foi innovation, seguida de sustainable development, sustainability e project management. Salienta-se a ocorrência dos termos ecodesign, design e life cycle que, segundo Dangelico (2016), são fatores de sucesso importantes para o desenvolvimento de produtos sustentáveis.

Com base no apresentado, tem-se que o caminho da sustentabilidade e de projetos com conceitos ambientais é o futuro das empresas, cabendo a elas adaptarem-se aos meios, caso contrário, estarão destinadas a desaparecer, pois de acordo com a Figura 3, o desenvolvimento de produtos inovadores com focos sustentáveis só tende a crescer e se disseminar por toda a sociedade. Além disso, espera-se que os consumidores respondam 
positivamente aos aumentos de preços que possam surgir com o desenvolvimento desses produtos, justificandose uma inovação perceptível e ao mesmo tempo educativa em prol do desenvolvimento sustentável.

Como sugestão para trabalhos futuros propõe-se uma análise adicional que aborde as técnicas que estão sendo utilizadas para o desenvolvimento de produtos inovadores e sustentáveis. Ademais, sobre as melhores práticas para o gerenciamento desses novos produtos e com uma investigação mais profunda, indica-se realizar uma pesquisa de mercado com os consumidores para saber como eles estão reagindo aos produtos desenvolvidos com esses novos conceitos.

\section{Referências}

Abedini, A., Rahman, R., Naeini, H. S. \& Ebrahim, N. A. (2017). The 100 most cited papers in industrial design: a bibliometric analysis. Exacta, 15(3), 515-526,

Abino, V., Balice, A., \& Dangelico, R. M. (2009). Environmental strategies and green product development: an overview on sustainability-driven companies. Business strategy and the environment, 18(2), 83-96.

Agyapong, F. O., Agyapong, A., \& Poku, K. (2017). Nexus between social capital and performance of micro and small firms in an emerging economy: The mediating role of innovation. Cogent Business \& Management, 4(1), 1309784.

Azarias, J. G., Coutinho, A. R., \& Campos, F. C. (2018). Análise bibliométrica sobre estudo de caso e Survey voltado a inteligência competitiva na indústria. Exacta, 16(4), 135-148.

Barros, A. J. da S., \& Lehfeld, N. A. de S. (2008). Fundamentos de Metodologia Científica. 3. ed. São Paulo: Person Education.

Berto, R. M. V. de S., \& Nakano, D. (2014). Revisitando a produção científica nos anais do Encontro Nacional de Engenharia de Produção. Production, 24(1), 225-232.

Bocken, N. M. P., Farracho, M., Bosworth, R., \& Kemp, R. (2014). The front-end of eco-innovation for ecoinnovative small and medium sized companies. Journal of Engineering and Technology Management, 31, 43-57.

Brones, F., Carvalho, M. M. de, \& Zancul, E. de S. (2014). Ecodesign in project management: a missing link for the integration of sustainability in product development?. Journal of Cleaner Production, 80, 106-118.

Carayannis, E., \& Grigoroudis, E. (2014). Linking innovation, productivity, and competitiveness: implications for policy and practice. The Journal of Technology Transfer, 39(2), 199-218.

Carvalho, S. W., Silvestre, B., \& Cunningham, P. (2017). Hitting the Nail on the Head! Insight into Consumer Assessment of Sustainability-Related Innovations. Long Range Planning, 50(6), 741-755.

Dangelico, R. M. (2016). Green product innovation: where we are and where we are going. Business Strategy and the Environment, 25(8), 560-576.

Dobrovolskienè, N., \& Tamošiūnienè, R. (2015). An index to measure sustainability of a business project in the construction industry: Lithuanian case. Sustainability, 8(1), 14.

Gerhardt, T. E., \& Silveira, D. T. (2009). Métodos de pesquisa. Porto Alegre: Plageder.

Gil, A. C. (2008). Métodos e técnicas de pesquisa social. 6. ed. Editora Atlas SA. 
Gmelin, H., \& Seuring, S. (2014). Achieving sustainable new product development by integrating product life-cycle management capabilities. International Journal of Production Economics, 154, 166-177.

Kahn, K. B., Barezak, G., Nicholas, J., Ledwith, A., \& Perks, H. (2012). An examination of new product development best practice. Journal of product innovation management, 29(2), 180-192.

Kechinski, C. P., Faccio, K., Rosa, L. R., \& Echeveste, M. E. S. (2010). Análise do modelo de desenvolvimento de produto de uma empresa fabricante de produtos e soluções para o setor automotivo. Exacta, 8(1), 81-88.

Luiz, J. V. R., Jugend, D., Jabbour, C. J. C., Luiz, O. R., \& Souza, F. B. de (2016). Ecodesign field of research throughout the world: mapping the territory by using an evolutionary lens. Scientometrics, 109, 241-259.

Martens, M. L., Kniess, C. T., Martens, C. D. P., \& Carvalho, M. M. (2016). Um estudo de inovação sustentável em projeto de desenvolvimento de produtos. Exacta, 14(3), 477-494.

Miguel, P. A. C. (Coord.). (2010). Metodologia de pesquisa em engenharia de produção e gestão de operações. Rio de Janeiro: Elsevier.

Moresi, E. (2003). Metodologia da pesquisa. Brasília: Universidade Católica de Brasília.

Morioska, S. N., \& Carvalho, M. M. de (2016). Sustentabilidade e gestão de projetos: um estudo bibliométrico. Production, 26(3), 656-674.

Pihlajamaa, M., Kaipia, R., Säilä, J., \& Tanskanen, K. (2017). Can supplier innovations substitute for internal R\&D? A multiple case study from an absorptive capacity perspective. Journal of Purchasing and Supply Management, 23(4), 242-255.

Theis, V., Haubert, B., Zmijewski, J., \& Schreiber, D. (2016). Gestão da inovação em uma empresa do setor de borracha e termoplástico. Exacta, 15(1), 15-29.

Van Eck, N. J., \& Waltman, L. Visualizing bibliometric networks. In Y. Ding, R. Rousseau, \& D. Wolfram (Eds.), Measuring scholarly impact: methods and practice (pp. 285-320). London: Springer.

Recebido em: 05 jul. 2018 / Aprovado em: 01 abr. 2019

Para referenciar este texto

Burin, H. P., Butzge, C., Silva, M. F. da., Moraes, J. A. R., \& Hoppe, D. A. (2020). Levantamento bibliométrico sobre o desenvolvimento de produtos inovadores e sustentáveis. Exacta, 18(2), 387-400. https://doi.org/10.5585/ExactaEP.v18n2.8879. 